\title{
Article
}

\section{Quantum Supertwistors}

\author{
Rita Fioresi ${ }^{1}$ and María Antonia Lledó ${ }^{2, * *(\text { ) }}$ \\ 1 Dipartimento di Matematica, Piazza Porta San Donato, 5 and FaBiT, Università di Bologna, \\ 40126 Bologna, Italy; fioresi@dm.unibo.it \\ 2 Departament de Física Teòrica, Universitat de València and IFIC(CSIC-UVEG), C/ Dr. Moliner, 50, \\ 46100 Burjassot, Spain \\ * Correspondence: Maria.Lledo@ific.uv.es
}

check for updates

Citation: Fioresi, R.; Lledó, M.A. Quantum Supertwistors. Symmetry 2021, 13, 1241. https://doi.org/ $10.3390 /$ sym 13071241

Academic Editor: Tajron Jurić

Received: 27 May 2021

Accepted: 7 July 2021

Published: 10 July 2021

Publisher's Note: MDPI stays neutral with regard to jurisdictional claims in published maps and institutional affiliations.

Copyright: (c) 2021 by the authors. Licensee MDPI, Basel, Switzerland. This article is an open access article distributed under the terms and conditions of the Creative Commons Attribution (CC BY) license (https:// creativecommons.org/licenses/by/ $4.0 /)$.

\begin{abstract}
In this paper, we give an explicit expression for a star product on the super-Minkowski space written in the supertwistor formalism. The big cell of the super-Grassmannian $\operatorname{Gr}(2|0,4| 1)$ is identified with the chiral, super-Minkowski space. The super-Grassmannian is a homogeneous space under the action of the complexification $\operatorname{SL}(4 \mid 1)$ of $\mathrm{SU}(2,2 \mid 1)$, the superconformal group in dimension 4 , signature $(1,3)$, and supersymmetry $N=1$. The quantization is done by substituting the groups and homogeneous spaces by their quantum deformed counterparts. The calculations are done in Manin's formalism. When we restrict to the big cell, we can explicitly compute an expression for the super-star product in the Minkowski superspace associated to this deformation and the choice of a certain basis of monomials.
\end{abstract}

Keywords: star products; superspace; non-commutative spacetime; quantum groups; quantum supergroups

\section{Introduction}

The Twistor theory [1-3] was initiated by Penrose as an alternative way of describing spacetime. One starts with an abstract, four-dimensional complex vector space ( twistor space) and the complex, compactified Minkowski space is the set of two planes inside the twistor space. This is the Grassmannian manifold $G(2,4)$, which is a homogeneous space of the group $\operatorname{SL}(4, \mathbb{C})$, that is, a homogeneous space of the group $\operatorname{SL}(4, \mathbb{C})$. This group is the spin group of the conformal group of spacetime, namely, $\mathrm{SU}(2,2)$.

One passes from the Minkowski space to a conformal space by a compactification, and vice versa by restricting to the big cell of the conformal space. Thus, one could think of a non-conformally-symmetric field theory as a conformal theory broken down to the big cell by some extra terms.

Conformal symmetry has a fundamental role in the gauge/gravity correspondence [4] (for a review, see $[5,6]$ ) which relates gravity theories to conformal gauge theories defined on the boundary of spacetime. It would then be interesting to see how conformal theories can be deformed and what the meaning of the deformation from the gravity point of view is.

In the original papers [1,2], Penrose believed that the Twistor theory could help to introduce the indetermination principle in spacetime. The points had to be 'smeared

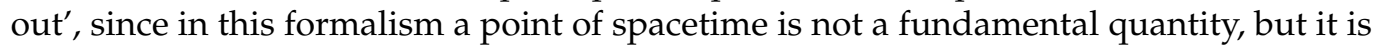
secondary to twistors.

Nevertheless, all the twistor constructions are classical. Our point of view is deforming the algebra of functions over spacetime to a non-commutative algebra. Because of the non-commutativity, this will introduce an indetermination principle among the coordinates of spacetime. A quantum group is a commutative, but non-cocommutative Hopf algebra depending on an indeterminate parameter $q$. One can specify $q=1$ to recover the original commutative Hopf algebra, which is just a Lie group. 
The quantum group $\mathrm{SL}_{q}(4, \mathbb{C})$ is the quantum conformal group complexified. The idea underlying the work of [7] was to make such a substitution and then to obtain a quantum Grassmannian, a quantum Minkowski space, and a quantum Poincaré group satisfying the same relations among them as their classical counterparts. The scheme is also generalized to flag manifolds. Thus, the quantum conformal group acts naturally on the quantum Grassmannian, viewed as a quantum homogeneous space, and the quantum Poincaré group is identified with the subgroup of it that preserves the big cell [7].

In the super-setting, we have several superspaces that are of interest: the Grassmannian supervariety $\operatorname{Gr}(2|0,4| 1)$, which corresponds in physical terms to the superalgebra of chiral superfields and the superflag $F l(2|0,2| 1,4 \mid 1)$, which is the compactification of the complexification of the $N=1$ Minkowski superspace. The same idea can be applied here with the supergroup SL(4|1) [8], which can also be deformed to a quantum supergroup. For a detailed treatment of all the super and non-super, classical, and quantum cases, see [9]. We will follow Manin's formalism [10] for quantum supergroups.

Here we deal with both cases, the super and non-super one. We have identified a quantization of the (super)conformal space as a homogeneous space of $\mathrm{SL}_{q}(4 \mid 1)$. In the big cell (the Minkowski (super)space), it can be presented as a concrete star product on the algebra of functions. There is an atlas of the Grassmannian with six identical cells, and the (super) star products in the intersections glue in such a way that one can recover the quantum Grassmannian.

Any conformal theory expressed in terms of twistors would have, presumably with this procedure, a quantum counterpart, where the word 'quantum' here means that we are deforming spacetime itself. The observables of such theories will be modified, but to compute the modifications explicitly it is not enough to look at the abstract algebra defined by generators and relations. One has to go to a 'semiclassical' approach where observables are still functions on spacetime as the original ones, but with a product that is non-commutative. This is achieved only if one has an explicit expression for the star product. In fact, the functions become (formal) series in the parameter of the deformation and there is no canonical choice for the star product among the equivalent ones. This problem is present in general in deformation quantization. For example, when one quantizes the phase space with a constant Poisson bracket, there seems to be a choice in which the star product has an easy computable expression, the Moyal-Weyl star product. Other than this simple case there are not, to the best of our knowledge, computations showing explicitly different star products, less being quadratic (at the leading order) deformations. To compute the star product one has to stick to a basis in the abstract algebra, and the explicit expression for the star product depends on such a choice. We have chosen here a sort of normal ordering, so the final expression does not have symmetry between the entries of the star product as the Moyal-Weyl has. It would have been more difficult to show the existence of a basis where the star product appears to be more symmetric, and then the calculation of the star product would have been more involved. For this reason, we have stuck to a normal ordering. There is one instance, though, were the ordering is not relevant and it is the deformation to order one in $\mathrm{h}\left(q=\mathrm{e}^{h}\right)$ of the commutator induced by the star product $f \star g-g \star f$. This is the Poisson bracket, that we also compute explicitly, with an expression far simpler than the star product itself. There is always an equivalent deformation where the Poisson bracket is the first term in the star product itself, so there is no need to antisymmetrize. Knowing the Poisson bracket would then be useful to compute first-order corrections to the theories at hand, induced by the non-commutativity of spacetime.

We first deal with the non-super case [11]. We work in the algebraic category, so we first give an explicit formula for the star product among two polynomials in the big cell of the Grassmannian. Since the quantum algebras that we present here are deformations of the algebra of polynomials on the Minkowski space, the star product that we obtain is also algebraic.

In the same reference [11] it is shown that this deformation can be extended to the set of smooth functions in terms of a differential star product. Since a differential operator is 
determined once it is given on polynomials, the bidifferential operators appearing in the star product are completely determined. The Poisson bracket leading the deformation is a quadratic one, so the Poisson structure is neither symplectic nor regular. For the super case we obtain that, at least to the first order in $h$, the differentiability property is maintained.

Examples of such transitions from the category of algebraic varieties to the category of differential manifolds in the quantum theory are given in [12-14]. There, the authors consider coadjoint orbits with the Kirillov-Kostant-Souriau symplectic form. It was shown that some algebraic star products do not have differential counterparts (not even modulo an equivalence transformation), so the results of [11] are non-trivial. It is remarkable that one of the algebraic star products that does not have a differential extension is the star product on the coadjoint orbits of SU(2), associated to the standard quantization of angular momentum. For algebraic star products and their classification, see also [15].

Other works also deal with the quantization of spacetime in terms of the twistor space. A very interesting work [16] applies the methods of geometric quantization to the twistor space.

The Weyl-Moyal star product is, in some sense, the simplest formal deformation that one can construct on $\mathbb{R}^{n}$. It requires a constant Poisson bracket:

$$
\{f(x), g(x)\}=B^{\mu v} \partial_{\mu} f(x) \partial_{\nu} g(x), \quad f, g \in C^{\infty}\left(\mathbb{R}^{n}\right),
$$

where $B_{\mu v}$ is any constant, antisymmetric matrix. The associative, non-commutative star product is given by $[17,18]$ :

$$
f \star g(x)=\sum_{n=0}^{\infty} \frac{h^{n}}{n !} B^{\mu_{1} v_{1}} \ldots B^{\mu_{n} v_{n}} \partial_{\mu_{1}} \ldots \partial_{\mu_{n}} f(x) \partial_{\nu_{1}} \ldots \partial_{v_{n}} g(x) .
$$

In [19], the Minkowski space is endowed, first, with a constant Poisson bracket as above. Then, using the R-matrix approach, the authors construct the action of the conformal group on the non-commutative space, which gives a deformation similar to the one used in [20].

The Moyal deformation of space-time has been used in String theory (the original references are [21,22]). In String theory, the presence of a $B_{\mu \nu}$ field with a non-zero vacuum expectation value can be interpreted as a deformation of space-time with the Moyal-Weyl deformation induced by $\left\langle B_{\mu \nu}\right\rangle$. This is a genuine non-commutative structure of spacetime. However, one has to take into account that it breaks the Lorentz invariance.

For the super case, there is also this type of 'Moyal-Weyl' deformation. It is known that the quantization of a Grassmann algebra is a Clifford algebra of split signature $(n, n)$.

There are very few deformations that can be given explicitly as a star product in closed form [23]. A general formula is known for an arbitrary Poisson bracket (Kontsevich's formula, [24]), but it is extremely hard to work out the coefficients for the differential operators appearing in the deformation, even for simpler, linear Poisson brackets. For many deformations, we only know how to express them in terms of generators and relations. While this may be enough from a mathematical point of view, it is often not enough for applications. The formula that we give is involved but it is explicit, and this is a real advantage.

In order to quantize the Grassmannian, one can also use the fact that the Grassmannian $G(m, n) \simeq \mathrm{SL}(n) / P$, with $\mathrm{P}$ a parabolic subgroup is, as a real manifold, a coadjoint orbit of the group $S L(n)$. In fact, any flag manifold is so, being the full flag $F l(1,2,3, \ldots, n)$, the regular (maximal dimension) orbit, and all the others non-regular. The approaches of $[12,13,25-27]$ would then be relevant here. The Kirillov-Kostant-Soriau Poisson bracket on the coadjoint algebra given essentially by the Lie bracket, is a linear Poisson bracket. It restricts to a symplectic Poisson bracket on the orbits. The star product is obtained from the enveloping algebra but it is only explicit once one takes symplectic coordinates on an open set of the orbit, in which case it is, locally, a Moyal-Weyl star product. It is then a star product equivariant under the action of the group. In the works mentioned above, the 
quantization is given in terms of generators and relations so it is an algebraic deformation, but then in [14] the relation with differential star products was studied. This mechanism could, in principle, be extended to the super case.

Another approach to the quantization of coadjoint orbits has been undertaken also in the Refs. [28-30] using the so-called it Shapovalov pairing of Verma modules.

Grassmannians have also been quantized as fuzzy spaces. A fuzzy space is built by using harmonic functions on the coset space and truncating the expansion at some level. The functions can be expressed as matrices in a certain basis and a product on the truncated space is defined just using matrix multiplication. We find this approach in [31,32].

We believe that the three approaches just mentioned must be linked in some way, since the quantizations are equivariant under the classical group $\operatorname{SL}(4, \mathbb{C})$ in this case) and all of them are intimately related to the representation theory. It is, however, not straightforward to compare them.

Interesting as these works are, our deformation is a different one. The Poisson bracket that we obtain on the Minkowski (super)space is a quadratic one (in particular, not symplectic) and the star product is then non-equivalent to a Weyl-Moyal one. Additionally, the equivariance of the star product is achieved only by deforming the group to a quantum group, contrary to the above-mentioned approaches. Nevertheless, we are able to give an explicit formula for it in terms of a recursive expression. The formulas for the non-super case are involved but manageable. For the super case, we have put the star product in terms of the non-super one, otherwise the notation becomes very heavy. This is an example of how a standard ordering in the generators of the quantum Minkowski (super)space induce a (super) star product that is not at all trivial. Contrary to many other deformations, whose algebra is given in terms of generators and relations, here we have an explicit (although involved) formula for the calculation of the star product of two monomials.

The organization of the paper is as follows:

In Section 2 we review the classical picture, also for the super case, and settle the notation for the algebraic approach. In Section 3 we describe the quantum super-Minkowski space obtained in the Reference [8], together with the corresponding quantum super groups. In Section 4 we tackle the even case studied in [11] and give the explicit formula for the star product between two polynomials on Minkowski space. We refer to that same paper to see how the differentiability of the star product is proven. Finally, in Section 5, based on the results obtained in the previous section, we obtain the star product for the super-Minkowski space.

For completeness, in Appendix A we have given a basis of the super Poincaré group in terms of its usual generators.

\section{The Classical Chiral Conformal and Minkowski Superspaces}

We describe here the $N=1$ chiral conformal superspace as the super-Grassmannian $\mathrm{Gr}:=\operatorname{Gr}(2|0,4| 1)$. The superspace $\mathrm{Gr}$ is a homogeneous superspace under the action of supergroup SL(4|1), which is the complexification of the conformal supergroup in Minkowskian signature, namely, $\mathrm{SU}(2,2 \mid 1)$. The chiral Minkowski superspace $\mathcal{M}$ is realized as the big cell inside Gr and the action of the Poincaré supergroup as the symmetries of Gr stabilizing $\mathcal{M}$.

For an explicit construction of this picture, see $[8,9,33,34]$. For the ordinary, non-super counterpart description, see also [3,11,33]. We briefly describe it here.

Let $\mathrm{Gr}_{0}=\operatorname{Gr}(2,4)$ denote the Grassmannian of 2-dimensional subspaces of $\mathbb{C}^{4}$. The space $\mathcal{T}_{0}=\mathbb{C}^{4}$ is called the twistor space. The Grassmannian $\operatorname{Gr}_{0}=\operatorname{Gr}(2,4)$ is a complex analytic manifold, a projective algebraic variety, and a homogeneous space under the action of the group $\operatorname{SL}(4, \mathbb{C})$. In fact, a two-dimensional subspace is given by two independent vectors:

$$
\pi=(a, b)=\left(\begin{array}{ll}
a_{1} & b_{1} \\
a_{2} & b_{2} \\
a_{3} & b_{3} \\
a_{4} & b_{4}
\end{array}\right)
$$


There is a natural right action of $\operatorname{SL}(2, \mathbb{C})$ corresponding to basis change. The left action of $\operatorname{SL}(4, \mathbb{C})$ is the obvious one and it is a transitive action. Selecting one element, say $\pi_{0}=\left(e_{1}, e_{2}\right)$ with $\left\{e_{i}\right\}_{i=1}^{4}$ the canonical basis in $\mathbb{C}^{4}$, we find that the isotropy group of $\pi_{0}$ is the upper parabolic subgroup

$$
P_{0}^{u}=\left\{\left(\begin{array}{cc}
L & M \\
0 & R
\end{array}\right) \in \operatorname{SL}(4, \mathbb{C}) \quad \mid \quad \operatorname{det} L \cdot \operatorname{det} R=1\right\},{ }^{\prime}
$$

where $R, L$ and $M$ are $2 \times 2$-matrices. Thus,

$$
\mathrm{Gr}_{0}=\mathrm{SL}(4, \mathbb{C}) / P_{0}^{u}
$$

The big cell of $\mathrm{Gr}_{0}$ is the set of points such that

$$
\operatorname{det}\left(\begin{array}{ll}
a_{1} & b_{1} \\
a_{2} & b_{2}
\end{array}\right) \neq 0
$$

By a right $\mathrm{SL}(2, \mathbb{C})$ transformation, we can bring (1) to the standard form

$$
U_{12}:=\left\{\left(\begin{array}{cc}
1 & 0 \\
0 & 1 \\
t_{31} & t_{32} \\
t_{41} & t_{42}
\end{array}\right)=\left(\begin{array}{l}
\mathbb{1} \\
t
\end{array}\right)\right\}
$$

with $t$ unconstrained. The big cell is then $U_{12} \approx \mathbb{C}^{4}$. The subset of $\operatorname{SL}(4, \mathbb{C})$ leaving the big cell invariant is the lower parabolic subgroup

$$
P_{0}^{l}=\left\{\left(\begin{array}{cc}
x & 0 \\
T x & y
\end{array}\right) \in \operatorname{SL}(4, \mathbb{C}) \quad \mid \quad \operatorname{det} x \cdot \operatorname{det} y=1\right\}
$$

where the unconstrained matrix $T x$ is written in this way to see the action better on the big cell. We have

$$
t \longrightarrow y t x^{-1}+T \text {. }
$$

Thus, $P_{0}^{l}$ is the Poincaré group including the dilations and the big cell is the Minkowski space, with its more familiar form $t=x^{\mu} \sigma_{\mu}$ in terms of the Pauli matrices. $P_{0}^{l}$ are

As algebraic groups, the coordinate algebras of $\mathrm{SL}_{4}:=\mathrm{SL}(4, \mathbb{C})$ and its subgroup

$$
\begin{array}{ll}
\mathbb{C}\left[\mathrm{SL}_{4}\right]=\mathbb{C}\left[g_{i j}\right] /(\operatorname{det} g-1), & i, j=1, \ldots, 4 \\
\mathbb{C}\left(P_{0}^{l}\right)=\mathbb{C}\left[x_{i j}, y_{k l}, T_{k j}\right] /(\operatorname{det} x \operatorname{det} y-1), & i, j=1,2, \text { and } k, l=3,4 .
\end{array}
$$

These algebras carry a well-known commutative Hopf algebra structure.

We can associate with $\mathrm{Gr}_{0}$ the $\mathbb{Z}$-graded ordinary algebra $\mathcal{O}\left(\mathrm{Gr}_{0}\right)$ given by its Plücker embedding in the projective space $\mathbf{P}^{5}$ (see, for example, [8,33] or [9] Ch. 2) in terms of six indeterminates $q_{i j}$ and the Plücker relation described below:

$$
\mathcal{O}\left(\mathrm{Gr}_{0}\right):=\mathbb{C}\left[q_{i j}\right] / \mathcal{I}_{P 0}, \quad 1 \leq i<j \leq 4,
$$

where $\mathcal{I}_{P 0}$ is the ideal generated by the Plücker relation:

$$
q_{12} q_{34}-q_{13} q_{24}+q_{14} q_{23}=0 .
$$

The interesting observation here, that will be key to obtain the quantization, is that $\mathcal{O}\left(\mathrm{Gr}_{0}\right)$ can be retrieved as a subalgebra of $\mathbb{C}\left[\mathrm{SL}_{4}\right]$. If we write the generators of $\mathbb{C}\left[\mathrm{SL}_{4}\right]$ in its usual matrix form 


$$
g=\left(\begin{array}{llll}
g_{11} & g_{12} & g_{13} & g_{14} \\
g_{21} & g_{22} & g_{23} & g_{24} \\
g_{31} & g_{32} & g_{33} & g_{34} \\
g_{41} & g_{42} & g_{43} & g_{44}
\end{array}\right)
$$

the determinants $d_{i j}=g_{i 1} g_{j 2}-g_{i 2} g_{j 1}$ with $1 \leq i<j \leq 4$, that is, all possible determinants of the two first columns, satisfy the Plücker relation (6) and this is the only independent relation that they satisfy (see [9]). Therefore,

$$
\mathcal{O}\left(\mathrm{Gr}_{0}\right) \cong \mathbb{C}\left[d_{i j}\right] \subset \mathbb{C}\left[\mathrm{SL}_{4}\right], \quad 1 \leq i<j \leq 4
$$

The condition (2) is related to the invertibility of $q_{12}$ in (6). Introducing $q_{12}^{-1}$ with $q_{12} q_{12}^{-1}-1=0$ and degree -1 , the subalgebra of $\mathcal{O}\left(\mathrm{Gr}_{0}\right)\left[q_{12}^{-1}\right]$ of degree 0 is the polynomial subalgebra freely generated by the elements

$$
\left(\begin{array}{ll}
t_{31} & t_{32} \\
t_{41} & t_{42}
\end{array}\right)=\left(\begin{array}{ll}
-d_{23} & d_{13} \\
-d_{24} & d_{14}
\end{array}\right) d_{12}^{-1}
$$

where $d_{i j}$ is the determinant formed by the elements of (7) in the positions of columns 1,2 and rows $i, j(i<j)$. The determinant $d_{34}$ can be obtained from (6). The calculation follows by taking the first two columns in (7) and multiplying on the right by

$$
\left(\begin{array}{ll}
g_{11} & g_{12} \\
g_{21} & g_{22}
\end{array}\right)^{-1}=\frac{1}{d_{12}}\left(\begin{array}{cc}
g_{22} & -g_{12} \\
-g_{21} & g_{11}
\end{array}\right)
$$

and then comparing with (3).

Similarly, we consider the $N=1$ supertwistor superspace, $\mathbb{C}^{4 \mid 1}$. We have the set Gr $:=\operatorname{Gr}(2|0,4| 1)$ of $2 \mid 0$ subspaces in $\mathbb{C}^{4 \mid 1}$. It is naturally an analytic supermanifold, a projective algebraic supervariety and a homogeneous superspace under the action of the supergroup SL(4|1). We will use the language of the functor of points (see, for example, $[9,34,35])$, better suited to treat supergroups. Thus, one has

$$
\operatorname{SL}(4 \mid 1)(\mathcal{A})=\left\{\left(\begin{array}{ll}
g_{i j} & \gamma_{i 5} \\
\gamma_{5 j} & g_{55}
\end{array}\right), \quad i, j=1, \ldots, 4\right\}
$$

where $\mathcal{A}$ is any superalgebra. The Latin letters will represent elements of $\mathcal{A}_{0}$, and the Greek ones, elements of $\mathcal{A}_{1}$, unless otherwise stated.

We can give an element in $\operatorname{Gr}(\mathcal{A})$, for $\mathcal{A}$ local, in terms of two even independent vectors

$$
\pi=(a, b)=\left(\begin{array}{ll}
a_{1} & b_{1} \\
a_{2} & b_{2} \\
a_{3} & b_{3} \\
a_{4} & b_{4} \\
\alpha_{5} & \beta_{5}
\end{array}\right)
$$

that, as before, can be chosen up to the right action of $\mathrm{SL}_{2}(\mathcal{A})$. The isotropy group of $\left\{e_{0}, e_{1}\right\}$, being $\left\{e_{i}, \epsilon_{5}, i=1, \ldots, 4\right\}$ the canonical basis, is the upper parabolic subgroup

$$
P^{u}(A)=\left\{\left(\begin{array}{ccc}
L & M & \alpha \\
0 & R & \beta \\
0 & \delta & d
\end{array}\right) \in \operatorname{SL}(4 \mid 1)(\mathcal{A}) \quad \mid \quad \operatorname{det} L \cdot \operatorname{det} R=d\right\},
$$

so

$$
\operatorname{Gr}(\mathcal{A})=\operatorname{SL}(4 \mid 1)(\mathcal{A}) / P^{u}(A) .
$$

We will say that $\mathrm{Gr}$ is the $N=1$ chiral superspace. 
The reduced manifold of $\mathrm{Gr}$ is $\operatorname{Gr}_{0}$, so the big cell of $\operatorname{Gr}(\mathcal{A})$ will be the set of $\mathcal{A}$ points with

$$
\operatorname{det}\left(\begin{array}{ll}
a_{1} & b_{1} \\
a_{2} & b_{2}
\end{array}\right) \text {, invertible, }
$$

which with a right $\mathrm{SL}_{2}(\mathcal{A})$ transformation, can be brought to the standard form

$$
\left(\begin{array}{cc}
1 & 0 \\
0 & 1 \\
t_{31} & t_{32} \\
t_{41} & t_{42} \\
\tau_{51} & \tau_{52}
\end{array}\right)=\left(\begin{array}{l}
11 \\
t \\
\tau
\end{array}\right) .
$$

We call the set of such matrices the big cell $U_{12}$; this is an open subsupermanifold of $\mathrm{Gr}$ and $U_{12} \cong \mathbb{C}^{4 \mid 2}$. The subgroup leaving the big cell invariant is the lower parabolic subgroup of SL(4|1):

$$
P^{l}=\left(\begin{array}{ccc}
x & 0 & 0 \\
T x & y & y \eta \\
d \rho & d \xi & d
\end{array}\right)
$$

and the action on the big cell is

$$
\left(\begin{array}{l}
\mathbb{1} \\
t^{\prime} \\
\tau^{\prime}
\end{array}\right)=\left(\begin{array}{c}
\mathbb{1} \\
y(t+\eta \tau) x^{-1}+T \\
d(\tau+\rho+\xi t) x^{-1}
\end{array}\right) .
$$

Taking $\xi=0$, we obtain the super Poincaré group including dilations. The condition $\xi=0$ will be necessary if we also consider the antichiral superspace $\operatorname{Gr}(2|1,4| 1)$.

There is a $\mathbb{Z}$-graded superalgebra associated with the super Plücker embedding of $\mathrm{Gr}$ in $\mathbf{P}^{6 \mid 4}$ (see [8,9] Ch. 4 for more details) given in terms of seven even indeterminates $q_{i j}, a_{55}$ and four odd indeterminates satisfying the relations described below:

$$
\mathcal{O}(\mathrm{Gr}):=\mathbb{C}\left[q_{i j}, \lambda_{k}, a_{55}\right] / \mathcal{I}_{P}, \quad i, j, k=1, \ldots, 4,
$$

where $I_{P}$ is the super ideal generated by the super Plücker relations:

$$
\begin{array}{ll}
q_{12} q_{34}-q_{13} q_{24}+q_{14} q_{23}=0, & \text { (classical Plücker relation) } \\
q_{i j} \lambda_{k}-q_{i k} \lambda_{j}+q_{j k} \lambda_{i}=0, & 1 \leq i<j<k \leq 4 \\
\lambda_{i} \lambda_{j}=a_{55} q_{i j} & 1 \leq i<j \leq 4 \\
\lambda_{i} a_{55}=0 . &
\end{array}
$$

As in the non-super case, we consider the first two rows in (8) and construct the quantities

$$
d_{i j}=g_{i 1} g_{j 2}-g_{i 2} g_{j 1}, \quad \sigma_{i}=g_{1 i} \gamma_{52}-g_{2 i} \gamma_{51}, \quad a=\gamma_{51} \gamma_{52},
$$

which satisfy the super Plücker relations and no other independent relations. In this way, one retrieves the algebra $\mathbb{C}\left[q_{i j}, \lambda_{i}, a_{55}\right] / \mathcal{I}_{P}$ of the super Plücker embedding as a subalgebra of SL(4|1).

The condition (10) defining the super-Minkowski space as the big cell in Gr is then equivalent to $q_{12} \neq 0$. The superalgebra of polynomials $\mathcal{O}(\mathcal{M})$ on $\mathcal{M}$ is then retrieved in $\mathcal{O}(\mathrm{Gr})$ as the elements of degree zero in $\mathcal{O}(\mathrm{Gr})\left[q_{12}^{-1}\right]$.

This subalgebra, $\mathcal{O}\left(U_{12}\right)=\mathcal{O}(\mathcal{M})$ is the polynomial subalgebra generated by the elements (see (11)) 


$$
\left(\begin{array}{ll}
t_{31} & t_{32} \\
t_{41} & t_{42} \\
\tau_{51} & \tau_{52}
\end{array}\right)=\left(\begin{array}{cc}
-d_{23} d_{12}^{-1} & d_{13} d_{12}^{-1} \\
-d_{24} d_{12}^{-1} & d_{14} d_{12}^{-1} \\
\sigma_{1} d_{12}^{-1} & \sigma_{2} d_{12}^{-1}
\end{array}\right)
$$

and the rest of the indeterminates can be obtained from these using the super Plücker relations (14) (Ref. [9] Ch. 5).

As supergroups, $\operatorname{SL}(4 \mid 1), P^{l}$, and also $P^{u}$ are super-Hopf algebras. This allows us to give the coaction of the relevant supergroup on the super-conformal or superMinkowski spaces.

\section{The Quantum Super-Minkowski Space}

We now briefly describe the quantization of super-Minkowski space obtained in [8], Section 2 (see also in [9] Ch. 5). We consider the quantum supergroup $\mathrm{SL}_{q}(4 \mid 1)$ in Manin's formalism [10]. In this section, we use the same letters for the classical and quantum generators, while it does not lead to confusion. We have the following definition:

Definition 1. The quantum matrix superalgebra $\mathrm{M}_{q}(m \mid n)$ is defined as

$$
\mathrm{M}_{q}(m \mid n)=_{\text {def }} \mathbb{C}_{q}\left\langle z_{i j}, \xi_{k l}\right\rangle / \mathcal{I}_{M},
$$

where $\mathbb{C}_{q}\left\langle z_{i j}, \xi_{k l}\right\rangle$ denotes the free superalgebra over $\mathbb{C}_{q}=\mathbb{C}\left[q, q^{-1}\right]$ generated by the even variables

$$
z_{i j}, \quad \text { for } \quad 1 \leq i, j \leq m \quad \text { or } \quad m+1 \leq i, j \leq m+n
$$

and by the odd variables

$$
\begin{aligned}
& \xi_{k l} \quad \text { for } \quad 1 \leq k \leq m, \quad m+1 \leq l \leq m+n \\
& \text { or } m+1 \leq k \leq m+n, \quad 1 \leq l \leq m,
\end{aligned}
$$

satisfying the relations $\tilde{\xi}_{k l}^{2}=0 . \mathcal{I}_{M}$ is an ideal generated by relations that we will describe shortly. We can visualize the generators as a matrix

$$
\left(\begin{array}{ll}
z_{m \times m} & \xi_{m \times n} \\
\xi_{n \times m} & z_{n \times n}
\end{array}\right)
$$

To simplify the notation, it is convenient sometimes to have a common notation for even and odd variables.

$$
a_{i j}= \begin{cases}z_{i j} & 1 \leq i, j \leq m, \text { or } \quad m+1 \leq i, j \leq m+n \\ \xi_{i j} & 1 \leq i \leq m, \quad m+1 \leq j \leq m+n, \text { or } \\ & m+1 \leq i \leq m+n, \quad 1 \leq j \leq m\end{cases}
$$

We assign a parity to the indices $p(i)=0$ if $1 \leq i \leq m$ and $p(i)=1$ otherwise. Then, the parity of $a_{i j}$ is $\pi\left(a_{i j}\right)=p(i)+p(j)$ mod 2 . Then, the ideal $\mathcal{I}_{M}$ is generated by the relations [10]:

$$
\begin{array}{ll}
a_{i j} a_{i l}=(-1)^{\pi\left(a_{i j}\right) \pi\left(a_{i l}\right)} q^{(-1)^{p(i)+1}} a_{i l} a_{i j}, & \text { for } j<l \\
a_{i j} a_{k j}=(-1)^{\pi\left(a_{i j}\right) \pi\left(a_{k j}\right)} q^{(-1)^{p(j)+1}} a_{k j} a_{i j}, & \text { for } i<k \\
a_{i j} a_{k l}=(-1)^{\pi\left(a_{i j}\right) \pi\left(a_{k l}\right)} a_{k l} a_{i j}, & \text { for } i<k, j>l \\
a_{i j} a_{k l}=(-1)^{\pi\left(a_{i j}\right) \pi\left(a_{k l}\right)} a_{k l} a_{i j}=(-1)^{\pi\left(a_{i j}\right) \pi\left(a_{k j}\right)}\left(q^{-1}-q\right) a_{k j} a_{i l}, & \text { or } i>k, j<l \\
& \text { for } i<k, j<l
\end{array}
$$


There is also a comultiplication

$$
\mathrm{M}_{q}(m \mid n) \stackrel{\Delta}{\longrightarrow} \mathrm{M}_{q}(m \mid n) \otimes \mathrm{M}_{q}(m \mid n)
$$

given formally by matrix multiplication, that is, $\Delta\left(a_{i j}\right)=\sum_{k} a_{i k} \otimes a_{k j}$.

Adjoining the inverse of the quantum Berezinian, which is a central element of the algebra, one has a suitable antipode map $S$, unit, and co-unit that define the standard quantum supergroup $\mathrm{GL}_{q}(4 \mid 1)$. One can restrict to $\mathrm{SL}_{q}(4 \mid 1)$ by setting the quantum Berezinian to 1 . We conveniently represent the generators in matrix form, as in (15)

$$
\left(\begin{array}{ll}
g_{i j} & \gamma_{i 5} \\
\gamma_{5 j} & g_{55}
\end{array}\right), \quad i, j=1, \ldots, 4
$$

As in the classical case, the quantum super-Poincaré group is given by

$$
\mathcal{O}_{q}(P):=\mathcal{O}\left(\mathrm{GL}_{q}(4 \mid 1)\right) / \mathcal{I}_{q},
$$

where $\mathcal{I}_{q}$ is the (two-sided) ideal in $\mathcal{O}\left(\mathrm{GL}_{q}(4 \mid 1)\right)$ generated by

$$
g_{1 j}, g_{2 j}, \quad \text { for } j=3,4 \text { and } \gamma_{15}, \gamma_{25}, \gamma_{53}, \gamma_{54} .
$$

We can equivalently write $\mathcal{O}_{q}(P)$ as generated, as in (12), by the following elements:

$$
\left(\begin{array}{ccc}
x & 0 & 0 \\
T x & y & y \eta \\
\rho x & 0 & d
\end{array}\right)
$$

with change of variables

$$
\begin{array}{ll}
x=\left(\begin{array}{ll}
g_{11} & g_{12} \\
g_{21} & g_{22}
\end{array}\right), & T=\left(\begin{array}{ll}
-q^{-1} D_{23} D_{12}^{-1} & D_{13} D_{12}^{-1} \\
-q^{-1} D_{24} D_{12}^{-1} & D_{14} D_{12}^{-1}
\end{array}\right), \\
y=\left(\begin{array}{ll}
g_{33} & g_{34} \\
g_{43} & g_{44}
\end{array}\right), & d=g_{55}, \\
\rho=\left(-q^{-1} D_{25} D_{12}^{-1}, D_{15} D_{12}^{-1}\right), & \eta=\left(\begin{array}{c}
-q^{-1} D_{34}^{34} D_{34}^{45} \\
D_{34}^{34-1} D_{34}^{35}
\end{array}\right) .
\end{array}
$$

We have denoted by $D_{i j}$ the determinant of the $i, j$ rows of the first two columns in (17) (see [9] Ch. 5 for more details).

In the following, it will be necessary to distinguish between (super)commutative and non-(super)commutative generators, so we put a hat " ' ' over the non-commutative ones. In the spirit of (15) we introduce the following definition:

Definition 2. The complexified quantum Minkowski superspace is the free algebra in six generators

$$
\begin{aligned}
& \hat{t}_{41}, \hat{t}_{42}, \hat{t}_{31} \text { and } \hat{t}_{32}, \quad \text { (even) } \\
& \hat{\tau}_{51}, \hat{\tau}_{52}
\end{aligned}
$$

satisfying the commutation relations 


$$
\begin{aligned}
& \hat{t}_{42} \hat{t}_{41}=q^{-1} \hat{t}_{41} \hat{t}_{42}, \\
& \hat{t}_{31} \hat{t}_{41}=q^{-1} \hat{t}_{41} \hat{t}_{31}, \\
& \hat{t}_{32} \hat{t}_{41}=\hat{t}_{41} \hat{t}_{32}+\left(q^{-1}-q\right) \hat{t}_{42} \hat{t}_{31}, \\
& \hat{t}_{31} \hat{t}_{42}=\hat{t}_{42} \hat{t}_{31}, \\
& \hat{t}_{32} \hat{t}_{42}=q^{-1} \hat{t}_{42} \hat{t}_{32}, \\
& \hat{t}_{32} \hat{t}_{31}=q^{-1} \hat{t}_{31} \hat{t}_{32},
\end{aligned}
$$

which would be the commutation relations defining the non-super quantum Minkowski space, together with

$$
\begin{array}{ll}
\hat{\tau}_{51} \hat{\tau}_{52}=-q^{-1} \hat{\tau}_{52} \hat{\tau}_{51} & \hat{t}_{32} \hat{\tau}_{52}=q^{-1} \hat{\tau}_{52} \hat{t}_{32} \\
\hat{t}_{31} \hat{t}_{51}=q^{-1} \hat{\tau}_{51} \hat{t}_{31} & \hat{t}_{42} \hat{\tau}_{52}=q^{-1} \hat{\tau}_{52} \hat{t}_{42} \\
\hat{t}_{41} \hat{\tau}_{51}=q^{-1} \hat{\tau}_{51} \hat{t}_{41}, & \hat{t}_{41} \hat{\tau}_{52}=\hat{\tau}_{52} \hat{t}_{41} \\
\hat{t}_{31} \hat{\tau}_{52}=\hat{\tau}_{52} \hat{t}_{31} & \hat{t}_{42} \hat{\tau}_{51}-\hat{\tau}_{51} \hat{t}_{42}=\left(q-q^{-1}\right) \hat{t}_{41} \hat{\tau}_{52} \\
\hat{t}_{32} \hat{\tau}_{51}=\hat{\tau}_{51} \hat{t}_{32}=\left(q-q^{-1}\right) \hat{t}_{31} \hat{\tau}_{52} &
\end{array}
$$

This algebra will be denoted as $\mathcal{O}_{q}(\mathcal{M})$, and it is a subalgebra of $\mathrm{M}_{q}(2 \mid 1)$ in Definition 1. If we denote the ideal given by (19) and (20) as $\mathcal{I}_{q \mathcal{M}}$, then we have that

$$
\mathcal{O}_{q}(\mathcal{M}) \equiv \mathbb{C}_{q}\left\langle\hat{t}_{41}, \hat{t}_{42}, \hat{t}_{31}, \hat{t}_{32}, \hat{\tau}_{51}, \hat{\tau}_{52}\right\rangle / \mathcal{I}_{q \mathcal{M}}
$$

The commutation relations of the generators of the quantum Poincare supergroup $\mathcal{O}\left(P_{q}\right) \mathcal{O}_{q}(P)(17),(18)$, are not trivial and are listed in [9] (pages 305-306) in a very similar notation. There is a natural coaction of $\mathcal{O}_{q}(P)$ on $\mathcal{O}_{q}(\mathcal{M})$, which is Proposition 7.5 of [8]. We give it here:

Proposition 1. The quantum chiral super-Minkowski space $\mathcal{O}_{q}(\mathcal{M})$ admits a coaction of the quantum chiral Poincaré supergroup $\mathcal{O}_{q}(P)$ :

$$
\begin{array}{r}
\mathcal{O}_{q}(\mathcal{M}) \stackrel{\hat{\Delta}}{\longrightarrow} \mathcal{O}_{q}(P) \otimes \mathcal{O}_{q}(\mathcal{M}) . \\
\hat{\Delta} \hat{t}_{i j}=t_{i j} \otimes \mathbb{1}+\hat{y}_{i a} S(\hat{x})_{b j} \otimes \hat{t}_{a b}+\hat{y}_{i} \hat{\eta}_{a} S(\hat{x})_{b j} \otimes \hat{\rho}_{j b}, \\
\hat{\Delta} \hat{\tau}_{j}=(\hat{d} \otimes \mathbb{1})\left(\hat{\tau}_{a} \otimes \mathbb{1}+\mathbb{1} \otimes \hat{\rho}_{a}\right)\left(S(\hat{x})_{a j} \otimes 1\right) .
\end{array}
$$

\section{The Star Product in the Even Case}

The star product allows us to recover the interpretation of the quantum algebra as the space of formal power series of standard polynomials, where a non-commutative product is defined. For the even case, the star product was computed in [11]. We will later make the generalization to the super case, but we first need to recall the construction in the non-super case.

Definition 3. The complexified quantum Minkowski space is the free algebra in four generators

$$
\hat{t}_{41}, \hat{t}_{42}, \hat{t}_{31} \text { and } \hat{t}_{32} \text {, }
$$

satisfying the relations (19).

This algebra will be denoted as $\mathcal{O}_{q}\left(\mathcal{M}_{0}\right)$. If we denote the ideal (19) by $\mathcal{I}_{\mathcal{M}_{0 q}}$, then we have that

$$
\mathcal{O}_{q}\left(\mathcal{M}_{0}\right) \equiv \mathbb{C}_{q}\left\langle\hat{t}_{41}, \hat{t}_{42}, \hat{t}_{31}, \hat{t}_{32}\right\rangle / \mathcal{I}_{\mathcal{M}_{0 q}}
$$


Let $\mathbb{C}_{q}=\mathbb{C}\left[q, q^{-1}\right]$. We have:

Proposition 2. There is an isomorphism $\mathcal{O}\left(\mathcal{M}_{0}\right)\left[q, q^{-1}\right] \approx \mathcal{O}_{q}\left(\mathcal{M}_{0}\right)$ as modules over $\mathbb{C}_{q}$. In fact, the map

$$
\begin{aligned}
\mathbb{C}_{q}\left[t_{41}, t_{42}, t_{31}, t_{32}\right] & \stackrel{Q_{\mathcal{M}_{0}}}{\longrightarrow} \mathcal{O}_{q}\left(\mathcal{M}_{0}\right) \\
t_{41}^{a} t_{42}^{b} t_{31}^{c} t_{32}^{d} & \longrightarrow \hat{t}_{41}^{a} \hat{t}_{42}^{b} \hat{t}_{31}^{c} \hat{t}_{32}^{d}
\end{aligned}
$$

is a module isomorphism (so it has an inverse).

Proof. The ordering used here is the normal ordering given in [10]. The Theorem 1.14 in this Reference says that such monomials are a basis for the algebra generated by the entries of the quantum matrix

$$
\left(\begin{array}{ll}
\hat{t}_{32} & \hat{t}_{31} \\
\hat{t}_{42} & \hat{t}_{41}
\end{array}\right)
$$

with commutation relations of the Manin relations (1) in the purely even, $n=2$ case. The proof of the proposition then follows.

A map like $Q_{\mathcal{M}_{0}}$ is called an ordering rule or quantization map. In particular, Proposition 2 is telling us that $\mathcal{O}_{q}\left(\mathcal{M}_{0}\right)$ is a free module over $\mathbb{C}_{q}$, with a basis of the set of standard monomials.

We can pull back the product on $\mathcal{O}_{q}\left(\mathcal{M}_{0}\right)$ to $\mathcal{O}\left(\mathcal{M}_{0}\right)\left[q, q^{-1}\right]$.

Definition 4. The star product on $\mathcal{O}\left(\mathcal{M}_{0}\right)\left[q, q^{-1}\right]$ is defined as

$$
f \star_{\text {even }} g=Q_{\mathcal{M}_{0}}^{-1}\left(Q_{\mathcal{M}_{0}}(f) Q_{\mathcal{M}_{0}}(g)\right), \quad f, g \in \mathcal{O}\left(\mathcal{M}_{0}\right)\left[q, q^{-1}\right] .
$$

By construction, the star product satisfies associativity. The algebra $\left(\mathcal{O}\left(\mathcal{M}_{0}\right)\left[q, q^{-1}\right], \star\right)$ is then isomorphic to $\mathcal{O}_{q}(\mathrm{M})$.

To give it explicitly [11], we first need a couple of partial results. We begin by computing some auxiliary relations.

Lemma 1. The following commutation rules are satisfied in $\mathcal{O}_{q}\left(\mathcal{M}_{0}\right)$ :

$$
\begin{aligned}
& \hat{t}_{42}^{m} \hat{t}_{41}^{n}=q^{-m n} \hat{t}_{41}^{n} \hat{t}_{42}^{m}, \\
& \hat{t}_{31}^{m} \hat{t}_{41}^{n}=q^{-m n} \hat{t}_{41}^{n} \hat{t}_{31}^{m}, \\
& \hat{t}_{31}^{m} \hat{t}^{n}=\hat{t}_{42}^{n} \hat{t}_{31}^{m}, \\
& \hat{t}_{32}^{m} \hat{t}_{42}^{n}=q^{-m n} \hat{t}_{42}^{n} \hat{t}_{32}^{m}, \\
& \hat{t}_{32}^{m} \hat{t}_{31}^{n}=q^{-m n} \hat{t}_{31}^{n} \hat{t}_{32}^{m},
\end{aligned}
$$

and

$$
\hat{t}_{32}^{m} \hat{t}_{41}^{n}=\hat{t}_{41}^{n} \hat{t}_{32}^{m}+\sum_{k=1}^{\mu} F_{k}(q, m, n) \hat{t}_{41}^{n-k} \hat{t}_{42}^{k} \hat{t}_{31}^{k} \hat{t}_{32}^{m-k},
$$

where $\mu=\min (m, n)$ and

$$
F_{k}(q, m, n)=\beta_{k}(q, m) \prod_{l=0}^{k-1} F(q, n-l) \text { with } F(q, n)=\left(\frac{1}{q^{2 n-1}}-q\right)
$$

and $\beta_{k}(q, m)$ is defined by the recursive relation

$$
\beta_{0}(q, m)=\beta_{m}(q, m)=1, \quad \text { and } \quad \beta_{k}(q, m+1)=\beta_{k-1}(q, m)+\beta_{k}(q, m) q^{-2 k} .
$$

Moreover, $\beta_{k}(q, m)=0$ if $k<0$ or if $k>m$. 
Proof. The proof is just a (lengthy) computation.

Theorem 1. The star product given in Definition 22 is given in two arbitrary monomials as

$$
\begin{aligned}
& \left(t_{41}^{a} t_{42}^{b} t_{31}^{c} t_{32}^{d}\right) \star_{\text {even }}\left(t_{41}^{m} t_{42}^{n} t_{31}^{p} t_{32}^{r}\right)=q^{-m c-m b-n d-d p} t_{41}^{a+m} t_{42}^{b+n} t_{31}^{c+p} t_{32}^{d+r}+ \\
& \mu=m i n(d, m) \\
& \sum_{k=1} q^{-(m-k) c-(m-k) b-n(d-k)-p(d-k)} F_{k}(q, d, m) . \\
& t_{41}^{a+m-k} t_{42}^{b+k+n} t_{31}^{c+k+p} t_{32}^{d-k+r}
\end{aligned}
$$

We consider now a change in the parameter, $q=\exp h$. The classic limit is then obtained as $h \rightarrow 0$. One can expand (24) in powers of $h$. In [11] it is shown that, at each order in $h$, the star product can be written as a bidifferential operator. Then the extension of the star product to $C^{\infty}$ functions is unique.

It is interesting to compute the antisymmetrization of the term of order 1 in $h$, which is the Poisson bracket

$$
\begin{aligned}
\{f, g\}_{\text {even }}= & t_{41} t_{31}\left(\partial_{41} f \partial_{31} g-\partial_{41} g \partial_{31} f\right)+t_{42} t_{41}\left(\partial_{41} f \partial_{42} g-\partial_{41} g \partial_{42} f\right)+ \\
& t_{32} t_{42}\left(\partial_{42} f \partial_{32} g-\partial_{42} g \partial_{32} f\right)+t_{32} t_{31}\left(\partial_{31} f \partial_{32} g-\partial_{31} g \partial_{32} f\right)+ \\
& 2 t_{42} t_{31}\left(\partial_{41} f \partial_{32} g-\partial_{41} g \partial_{32} f\right) .
\end{aligned}
$$

We can express the Poisson bracket in terms of the usual variables in the Minkowski space. The coordinate change is

$$
\left(\begin{array}{ll}
t_{31} & t_{32} \\
t_{41} & t_{42}
\end{array}\right)=x^{\mu} \sigma_{\mu}=\left(\begin{array}{cc}
x^{0}+x^{3} & x^{1}-\mathrm{i} x^{2} \\
x^{1}+\mathrm{i} x^{2} & x^{0}-x^{3}
\end{array}\right)
$$

and the inverse change is

$$
x^{0}=\frac{1}{2}\left(t_{31}+t_{42}\right), \quad x^{1}=\frac{1}{2}\left(t_{32}+t_{41}\right), \quad x^{2}=\frac{i}{2}\left(t_{32}-t_{41}\right), \quad x^{3}=\frac{1}{2}\left(t_{31}-t_{42}\right) .
$$

In these variables, the Poisson bracket becomes

$$
\begin{aligned}
\{f, g\}_{\text {even }}= & \mathrm{i}\left(\left(\left(x^{0}\right)^{2}-\left(x^{3}\right)^{2}\right)\left(\partial_{1} f \partial_{2} g-\partial_{1} g \partial_{2} f\right)+x^{0} x^{1}\left(\partial_{0} f \partial_{2} g-\partial_{0} g \partial_{2} f\right)-\right. \\
& x^{0} x^{2}\left(\partial_{0} f \partial_{1} g-\partial_{0} g \partial_{1} f\right)-x^{1} x^{3}\left(\partial_{2} f \partial_{3} g-\partial_{2} g \partial_{3} f\right)+ \\
& \left.x^{2} x^{3}\left(\partial_{1} f \partial_{3} g-\partial_{1} g \partial_{3} f\right)\right) .
\end{aligned}
$$

The Poisson bracket that we obtain is quadratic in the variables, and it is non-trivial.

\section{The Super-Star Product}

We want to repeat the procedure of Section 4 . We start by giving an ordering which defines a quantization map.

Proposition 3. There is an isomorphism

$$
\mathcal{O}(\mathcal{M})\left[q, q^{-1}\right]=\mathbb{C}_{q}\left[\tau_{51}, \tau_{52}, t_{41}, t_{42}, t_{31}, t_{32}\right] \approx \mathcal{O}_{q}(\mathcal{M})
$$

as modules over $\mathbb{C}_{q}$. In fact, for $a, b, c, d=0,1,2 \ldots$ and $e, f=0,1$ the map

$$
\begin{gathered}
\mathcal{O}(\mathcal{M})\left[q, q^{-1}\right] \stackrel{Q_{\mathcal{M}}}{\longrightarrow} \begin{array}{c}
\mathcal{O}_{q}(\mathcal{M}) \\
\tau_{51}^{e} \tau_{52}^{f} t_{41}^{a} t_{42}^{b} t_{31}^{c} t_{32}^{d}
\end{array} \hat{\tau}_{51}^{e} \hat{\tau}_{52}^{f} \hat{t}_{41}^{a} \hat{t}_{42}^{b} \hat{t}_{31}^{c} \hat{t}_{32}^{d}
\end{gathered}
$$

is a module isomorphism (so it has an inverse). 
Proof. The ordering used here is the normal ordering used in Theorem 1.14 of [10].

For the even part, we are in the situation of Proposition (2). Adding the odd variables in that reference says that such monomials are a basis for the algebra generated by the entries of the (non-square) quantum matrix

$$
\left(\begin{array}{ll}
\hat{t}_{32} & \hat{t}_{31} \\
\hat{t}_{42} & \hat{t}_{41} \\
\hat{\tau}_{52} & \hat{\tau}_{51}
\end{array}\right)
$$

with commutation relations the Manin relations (1). To add the odd variables, one has to check that they do not introduce other generators with the commutation relations, the Manin relations (1). These relations are the same (up to a sign) for even or odd generators. Hence, the normal ordering in Theorem 1.14 of [10] will give us the result using the same argument as in Prop. 2.

We first need a couple of partial results:

Lemma 2. For $a, b, c, d=0,1,2, \ldots$ we have the following commutation relations:

$$
\begin{aligned}
& \hat{t}_{41}^{a} \hat{\tau}_{52}=q^{-a} \hat{\tau}_{52} \hat{t}_{41}^{a} \\
& \hat{t}_{42}^{b} \hat{\tau}_{52}=q^{-b} \hat{\tau}_{52} \hat{t}_{42}^{b} \\
& \hat{t}_{31}^{c} \hat{\tau}_{52}=\hat{\tau}_{52} \hat{t}_{31}^{c} \\
& \hat{t}_{41}^{a} \hat{\tau}_{51}=q^{-a} \hat{\tau}_{51} \hat{t}_{41}^{a} \\
& \hat{t}_{42}^{b} \hat{\tau}_{51}=\hat{\tau}_{51} \hat{t}_{42}^{b}+\left(q-q^{-2 b+1}\right) \tau_{52} t_{41} t_{42}^{b-1} \\
& \hat{t}_{31}^{c} \hat{\tau}_{51}=q^{-c} \hat{\tau}_{51} \hat{t}_{31}^{c} \\
& \hat{t}_{32}^{d} \hat{\tau}_{51}=\hat{\tau}_{51} \hat{t}_{32}^{d}+\left(q-q^{-2 d+1}\right) \tau_{52} t_{31} t_{32}^{d-1} \\
& \hat{t}_{32}^{d} \hat{\tau}_{52}=q^{-d} \hat{\tau}_{52} \hat{t}_{32}^{d}
\end{aligned}
$$

Lemma 3. For $a, b, c, d=0,1,2, \ldots$

$$
\begin{aligned}
& t_{41}^{a} t_{42}^{b} t_{31}^{c} t_{32}^{d} \tau_{51}=q^{-(a+c)} \tau_{51} t_{41}^{a} t_{42}^{b} t_{31}^{c} t_{32}^{d}+q^{-(a+c)}\left(q-q^{-2 b+1}\right) \tau_{52} t_{41}^{a+1} t_{42}^{b-1} t_{31}^{c} t_{32}^{d}+ \\
& q^{-(a+b)}\left(q-q^{-2 d+1}\right) \tau_{52} t_{41}^{a} t_{42}^{b} t_{31}^{c+1} t_{32}^{d-1} \\
& t_{41}^{a} t_{42}^{b} t_{31}^{c} t_{32}^{d} \tau_{52}=q^{-(a+b+d)} \tau_{52} t_{41}^{a} t_{42}^{b} t_{31}^{c} t_{32}^{d}
\end{aligned}
$$

In order to simplify the notation, we define

$$
T(a, b, c, d)=t_{41}^{a} t_{42}^{b} t_{31}^{c} t_{32}^{d} .
$$

As in the non-super case, we have:

Definition 5. The super-star product on $\mathcal{O}\left(\mathcal{M}_{0}\right)\left[q, q^{-1}\right]$ is defined as

$$
f \star g=Q_{\mathcal{M}}^{-1}\left(Q_{\mathcal{M}}(f) Q_{\mathcal{M}}(g)\right), \quad f, g \in \mathcal{O}(\mathcal{M})\left[q, q^{-1}\right] .
$$

Theorem 2. The super-star product of two monomials in the given basis

$$
S=\tau_{51}^{e} \tau_{52}^{f} T(a, b, c, d) \star \tau_{51}^{u} \tau_{52}^{v} T(m, n, p, r),
$$

with $e, f, u, v=0,1$ and $a, b, c, d, m, n, p, r=0,1,2, \ldots$ is given, in terms of the even star product by (22) (the exponents of the odd variables are always taken $\bmod (2)$, so they take values 0 or 1 ): 


$$
\begin{aligned}
S= & \delta_{u 0} \delta_{v 0} \tau_{51}^{e} \tau_{52}^{f} T(a, b, c, d) \star_{\text {even }} T(m, n, p, r)+ \\
& \delta_{u 0} \delta_{v 1}\left(q^{-(a+b+d)} \tau_{51}^{e} \tau_{52}^{f+1} T(a, b, c, d) \star_{\text {even }} T(m, n, p, r)\right) \\
& \delta_{u 1} \delta_{v 0}\left((-1)^{f} q^{f-a-c} \tau_{51}^{e+1} \tau_{52}^{f} T(a, b, c, d) \star_{\text {even }} T(m, n, p, r)+\right. \\
& q^{-a-c}\left(q-q^{-2 b+1}\right) \tau_{51}^{e} \tau_{52}^{f+1} T(a+1, b-1, c, d) \star_{\text {even }} T(m, n, p, r)+ \\
& \left.q^{-a-b}\left(q-q^{-2 d+1}\right) \tau_{51}^{e} \tau_{52}^{f+1} T(a, b, c+1, d-1) \star_{\text {even }} T(m, n, p, r)\right)+ \\
& \delta_{u 1} \delta_{v 1}\left((-1)^{f} q^{-2 a-b-c-d+f} \tau_{51}^{e+1} \tau_{52}^{f+1} T(a, b, c, d) \star_{\text {even }} T(m, n, p, r)+\right. \\
& q^{-2 a-b-c-d+1)}\left(q-q^{-2 b+1}\right) \tau_{51}^{e} \tau_{52}^{f} T(a+1, b-1, c, d) \star_{\text {even }} T(m, n, p, r)+ \\
& \left.q^{-2 a-2 b-d+1}\left(q-q^{-2 d+1}\right) \tau_{51}^{e} \tau_{52}^{f} T(a, b, c+1, d-1) \star_{\text {even }} T(m, n, p, r)\right),
\end{aligned}
$$

where the star products between the Ts are given in Theorem 1. It is a quantum deformation of $\mathcal{O}(\mathcal{M})$.

Proof. For the proof, we have to reorder according to 3 . It is easy to check that for $q=1$, one obtains the standard, (super)commutative product on $\mathcal{O}(\mathcal{M})$.

The antisymmetrization of the first order in $h$ of the star product gives the Poisson bracket. To make the notation even lighter, we will write

$$
\begin{array}{ll}
R_{A}=\tau_{51}^{e} \tau_{52}^{f} t_{41}^{a} t_{42}^{b} t_{31}^{c} t_{32}^{d}, & R_{M}=\tau_{51}^{u} \tau_{52}^{v} t_{41}^{m} t_{42}^{n} t_{31}^{r} t_{32}^{p}, \\
R_{A}^{+}=\tau_{51}^{e} \tau_{52}^{f} t_{41}^{a+1} t_{42}^{b-1} t_{31}^{c} t_{32}^{d}, & R_{A}^{-}=\tau_{51}^{e} \tau_{52}^{f} t_{41}^{a} t_{42}^{b} t_{31}^{c+1} t_{32}^{d-1},
\end{array}
$$

when there is no possibility of confusion. Let us denote $S_{1}\left(R_{A}, R_{M}\right)$ the term of order $h$ in (28). We denote by $C_{1}$ the same term in (22).

$$
\begin{aligned}
S_{1}\left(R_{A}, R_{M}\right)= & C_{1}\left(R_{A}, R_{M}\right)+\delta_{u 0} \delta_{v 1}\left(-(a+b+d) R_{A} R_{M}\right) \\
& \delta_{u 1} \delta_{v 0}\left((f-a-c) R_{A} R_{M}+2 b R_{A}^{+} R_{M}+2 d R_{A}^{-} R_{M}\right) \\
& \delta_{u 1} \delta_{v 1}\left((-2 a-b-c+f) R_{A} R_{M}+2 b R_{A}^{+} R_{M}+2 d R_{A}^{-} R_{M}\right) .
\end{aligned}
$$

We now take into account that for $u, v=0,1$

$$
\begin{array}{ll}
\delta_{u 0}=1-u, & \delta_{v 0}=1-v, \\
\delta_{u 1}=u, & \delta_{v 1}=v .
\end{array}
$$

Then the terms proportional to $u v$ in (29) cancel out, so we are left with

$$
\begin{aligned}
S_{1}\left(R_{A}, R_{M}\right)= & C_{1}\left(R_{A}, R_{M}\right)-(a+b+d) R_{A} \cdot v R_{M}+(f-a-c) R_{A} \cdot u R_{M}+ \\
& 2 b R_{A}^{+} \cdot u R_{M}+2 d R_{A}^{-} \cdot u R_{M} .
\end{aligned}
$$

This expression can be written in terms of differential operators. We have, for example, that

$$
a R_{A}=t_{41} \partial_{t_{41}} R_{A}, \quad b R_{A}^{+}=t_{41} \partial_{t_{42}} R_{A}, \quad u R_{M}=\tau_{51} \partial_{\tau_{51}} R_{M}, \ldots
$$

The result is

$$
\begin{aligned}
S_{1}\left(R_{A}, R_{M}\right)= & C_{1}\left(R_{A}, R_{M}\right)+\left(t_{41} \partial_{t_{41}}+t_{42} \partial t_{42}+t_{32} \partial_{t_{32}}\right) R_{A} \cdot \tau_{52} \partial_{\tau_{52}} R_{M}+ \\
& \left(-\tau_{52} \partial_{\tau_{52}}-t_{41} \partial_{t_{41}}-t_{31} \partial_{t_{31}}+2 t_{41} \partial_{t_{42}}+2 t_{31} \partial_{t_{32}}\right) R_{A} \cdot \tau_{51} \partial_{\tau_{51}} R_{M},
\end{aligned}
$$


which satisfies the Leibniz rule in both arguments. Its (anti)symmetrization is the Poisson bracket

$$
\left\{R_{A}, R_{M}\right\}=S_{1}\left(R_{A}, R_{M}\right)-(-1)^{p_{A} p_{M}} S_{1}\left(R_{M}, R_{A}\right) .
$$

At this order, the star product is differential. Presumably, it will be differential at all orders, as its non-super counterpart [11].

Author Contributions: Both authors have had similar roles in the development of this research and the writing of the article. All authors have read and agreed to the published version of the manuscript.

Funding: This research was funded by Ministerio de Economía y Competitividad (Spain) grant number FIS2017-84440-C2-1-P and Generalitat Valenciana, grant number PROMETEO/2020/079.

Conflicts of Interest: The authors declare no conflict of interest. The funders had no role in the design of the study; in the collection, analyses, or interpretation of data; in the writing of the manuscript, or in the decision to publish the results.

\section{Appendix A. A Basis for the Poincaré Quantum Supergroup}

In this appendix, we give a brief sketch of the fact that the ordered monomials-according to a given ordering - of the generators super of the Poincaré quantum group form a basis for its quantum superalgebra. This is a non-trivial result based on the classical work by Bergman [36], but it is a modification of the argument in [11].

We recall the following key result.

Theorem A1. (Diamond Lemma). Let $R$ be the ring defined by generators and relations as:

$$
R:=\mathbb{C}_{q}\left\langle x_{i}\right\rangle /\left(X_{I_{k}}-f_{k}, k=1 \ldots s\right) .
$$

If $\Pi=\left\{X_{I_{k}}, f_{k}\right\}_{k=1, \ldots, s}$ is compatible with the ordering $<$ and all ambiguities are resolvable, then the set of ordered monomials $\Pi$ is a basis for $R$. Hence, $R$ is a free module over $\mathbb{C}_{q}$.

Let us fix a total order $\mathcal{O}$ on the variables $x, y, t, \tau$ as follows:

$$
\begin{aligned}
& \tau_{52}>\tau_{51}>t_{32}>t_{31}>t_{42}>t_{41}> \\
& x_{11}>x_{12}>x_{21}>x_{22}>y_{33}>y_{34}>y_{43}>y_{44} .
\end{aligned}
$$

Theorem A2. Let $\mathcal{O}_{q}(P)=\mathbb{C}_{q}\left\langle x_{i j}, y_{k l}, t_{i l}\right\rangle / \mathcal{I}_{P}$ be the algebra corresponding to the quantum Poincaré group. Then, the monomials in the order $\mathcal{O}$ as above are a basis for $\mathcal{O}_{q}(P)$.

The proof of this result is completely analogous to the non-super setting. It is an application of the Diamond Lemma, where we resolve all ambiguities using the Manin relations, whose form, in fact, does not depend on the generators having parity.

\section{References}

1. Penrose, R. Twistor algebra. J. Math. Phys. 1967, 8, 345-366. [CrossRef]

2. Penrose, R.; MacCallum, M.A.H. Twistor theory: An approach to the quantisation of fields and space-time. Phys. Rep. 1972, 6, 241-316. [CrossRef]

3. Ward, R.S.; Wells, R.O., Jr. Twistor Geometry and Field Theory; Cambridge University Press: Cambridge, UK, 1990.

4. Maldacena, J.M. The Large N limit of superconformal field theories and supergravity. Int. J. Theor. Phys. 1999, 38, 1113-1133. [CrossRef]

5. Maldacena, J.M. The Gauge/gravity duality. arXiv 2011, arXiv:1106.6073.

6. Ammon, M.; Erdmenger, J. Gauge/Gravity Duality. Foundations and Applications; Cambridge University Press: Cambridge, UK, 2015.

7. Fioresi, R. Quantum deformation of the flag variety. Commun. Algebra 1999, 27, 5669-5685. [CrossRef]

8. Cervantes, D.; Fioresi, R.; Lledó, M. The quantum chiral Minkowski and conformal superspaces. Adv. Theor. Math. Phys. 2011, 15, 565-620. [CrossRef]

9. Fioresi, R.; Lledó, M.A. The Minkowski and Conformal Superspaces: The Classical and Quantum Descriptions; World Scientific: Singapore, 2015. 
10. Manin, Y. Multiparametric quantum deformation of the general linear supergroup. Comm. Math. Phys. 1989, 123, 163-175. [CrossRef]

11. Cervantes, D.; Fioresi, R.; Lledó, M.A.; Nadal., F.A. Quantum twistors. P-Adic Numbers Ultrametric Anal. Appl. 2016, 8, 2-30. [CrossRef]

12. Fioresi, R.; Lledó, M.A. On the deformation quantization of coadjoint orbits of semisimple Lie groups. Pac. J. Math. 1999, 198, 411-436. [CrossRef]

13. Lledó, M.A. Deformation quantization of nonregular orbits of compact Lie groups. Lett. Math. Phys. 2001, 58, 57-67. [CrossRef]

14. Fioresi, R.; Levrero, A.; Lledó, M. A. Algebraic and differential star products on regular orbits of compact Lie groups. Pac. J. Math. 2002, 206, 321-337. [CrossRef]

15. Kontsevich, M. Deformation quantization of algebraic varieties. Lett. Math. Phys. 2006, 56, 271-294. [CrossRef]

16. Carey, A.L.; Hannabuss, K.C. Twistors an geometric Quantization. Rep. Math. Phys. 1978, 13, 199-231. [CrossRef]

17. Weyl, H. The Theory of Groups and Quantum Mechanics; Dover: New York, NY, USA, 1931.

18. Moyal, J.E. Quantum mechanics as a statistical theory. In Mathematical Proceedings of the Cambridge Philosophical Society; Cambridge University Press: Cambridge, UK, 1949; Volume 45, p. 99.

19. Hannabuss, K.C. non-commutative twistor space. Lett. Math. Phys. 2001, 58, 153-166. [CrossRef]

20. Kapustin, A.; Kuznetsov, A.; Orlov, D. non-commutative instantons and twistor transform. Comm. Math. Phys. 2001, 221, 385-432. [CrossRef]

21. Connes, A.; Douglas, M.R.; Schwarz, A.S. non-commutative geometry and matrix theory: Compactification on tori. JHEP 1998, 9802, 3. [CrossRef]

22. Seiberg, N.; Witten, E. String theory and non-commutative geometry. JHEP 1999, 9909, 32. [CrossRef]

23. Bayen, F.; Flato, M.; Fronsdal, C.; Lichnerowicz, A.; Sternheimer, D. Deformation theory and quantization, 1. Deformations of symplectic structures. Ann. Phys. 1978, 111, 61. [CrossRef]

24. Kontsevich, M. Deformation quantization of Poisson manifolds. Lett. Math. Phys. 2003, 66, 157-216. [CrossRef]

25. Cahen, M.; Gutt, S. Produits * sur les Orbites des Groupes Semi-Simples de Rang 1. C.R. Acad. Sc. Paris 1983, $296,821-823$.

26. Cahen, M.; Gutt, S.; Rawnsley, J. On Tangential Star Products for the Coadjoint Poisson Structure. Comm. Math. Phys. 1996, 180, 99-108. [CrossRef]

27. Cahen, M.; Gutt, S.; Rawnsley, J. Quantization of Kaehler manifolds I to IV. Trans. Am. Math. Thus,c. 1993, 337, 73-98.

28. Alekseev, A.; Lachowska, A. Invariant *-products on coadjoint orbits and the Shapovalov pairing. Comment. Math. Helv. 2005, 80, 795-810. [CrossRef]

29. Enriquez, B.; Etingof, P.; Marshall, I. Quantization of some Poisson-Lie dynamical r-matrices and Poisson homogeneous spaces. Contemp. Math. 2007, 433, 135-175.

30. Mudrov, A. Orthogonal basis for the Sahpovalov form of $\mathrm{U}_{q}(s l(n+1))$. Rev. Math. Phys. 2015, 27, 1550004. [CrossRef]

31. Donin, J.; Mudrov, A. Explicit equivariant quantization on coadjoint orbits of GL(n, $\mathbb{C})$. Lett. Math. Phys. 2002, 62, 17-32. [CrossRef]

32. Dolan, B.P.; Jahn, O. Fuzzy complex Grassmannian spaces and their star products. Int. J. Mod. Phys. A 2003, 18, 1935-1958. [CrossRef]

33. Varadarajan, V.S. Supersymmetry for Mathematicians: An Introduction; Courant Lecture Notes; AMS: Providence, RI, USA, 2004; Volume 1.

34. Carmeli, C.; Caston, L.; Fioresi, R.; Dimitrov, I. Mathematical Foundation of Supersymmetry; European Mathematical Society: Zurich, Germany, 2011.

35. Deligne, P.; Morgan, J. Notes on supersymmetry (following J. Bernstein). In Quantum Fields and Strings. A Course for Mathematicians; AMS: Providence, RI, USA, 1999; Volume 1.

36. Bergman, G. The diamond lemma for ring theory. Adv. Math. 1978, 29, 178-218. [CrossRef] 